\title{
Perioperative Respiratory Adverse Events in General Anesthesia Among Pediatric Surgical Patients in Comprehensive Specialized Hospitals in Northwest Ethiopia, 2020; A Cross-Sectional Follow-Up
}

Yophtahe Woldegerima ( $\nabla$ yophtii@gmail.com )

University Of Gondar

Desalegn Muche

Amhara Regional Health Bureau

Wubie Birlie

University Of Gondar

Habtu Adane

University Of Gondar

Misganaw Mengie

University Of Gondar

\section{Research Article}

Keywords: Perioperative Respiratory Adverse Events, Perioperative complications, Pediatric Anesthesia General Anesthesia

Posted Date: January 14th, 2021

DOl: https://doi.org/10.21203/rs.3.rs-138101/v1

License: (c) (1) This work is licensed under a Creative Commons Attribution 4.0 International License.

Read Full License

Version of Record: A version of this preprint was published at Frontiers in Pediatrics on February 11th, 2022. See the published version at https://doi.org/10.3389/fped.2022.827663. 
Perioperative respiratory adverse events in general anesthesia among pediatric surgical patients in Comprehensive Specialized Hospitals in Northwest Ethiopia, 2020; a crosssectional follow-up

Desalegn Muche Wudineh ${ }^{1}$, Yophtahe Woldegerima Berhe²*, Wubie Birlie Chekol², Habtu Adane², Misganaw Mengie Workie ${ }^{2}$

1 = Department of Anesthesia, Felege-Hiwot Referral Hospital, Bahirdar, Amhara National Regional State, Ethiopia

${ }^{2}=$ Department of Anesthesia, College of Medicine and Health Sciences, University of Gondar, Gondar, Ethiopia

$*=$ the corresponding author

The Addresses of the corresponding author: Yophtahe Woldegerima Berhe (2*), Department of Anesthesia, College of Medicine and Health Sciences, University of Gondar, Gondar, Ethiopia.

Email:yophtii@gmail.com

Phone: +251-912-494282

P.O.Box: 196 


\section{Abstract}

Introduction: Perioperative respiratory adverse events (PRAEs) are the most frequent complications in pediatrics which frequently result in morbidity and mortality. They are accountable for $75 \%$ of perioperative critical incidents and 33\% of cardiac arrests. The occurrence and severity of PRAEs depends on the natures of surgery, anesthesia \& patient's status.

Objective: To assess the incidence and factors associated with PRAEs in general anesthesia among pediatric surgical patients at the University of Gondar and Tibebe-Ghion Comprehensive Hospital and Specialized Hospitals, Northwest Ethiopia, 2020.

Methods: After obtaining the ethical approval, a hospital-based prospective cross-sectional follow-up study was conducted among pediatric surgical patients who underwent variety of surgical operations. A total of 225 patients were included. The associations between independent variables and the outcome variables were determined at $95 \%$ CI with the Chi-squared test, Fisher-exact test, bivariate, and multivariate logistic regression. Hosmer-Lemeshow test was used to assess the goodness of fit. Variables with a p-value $<0.05$ were considered significant.

Results: The incidence of PRAEs among 210 (93.3\% response rate) pediatrics surgical patients was $26.2 \%$ (95\% CI: 20.5, 30.9). A total of 129 episodes of PRAEs were observed. Most of the adverse events (89 $(69.0 \%))$ were occurred postoperatively. Desaturation was found to be the predominant adverse event which was occurred 61 (47.3\%) times. Age < 1 year (AOR: 3.6, 95\% CI: 1.3, 10.0), ASA $\geq 3$ (AOR: 5.2, 95\% CI: 1.9, 22.9), upper respiratory tract infections (AOR: 7.6, 95\% CI: 1.9, 30.2), presence of secretions in the upper airway (AOR: 4.8, 95\% CI: 1.4, 15.9) and airway related procedures (AOR: 6.0, 95\% CI: 1.5, 24.1) were significantly associated PRAEs.

Conclusions: The incidence of PRAEs among pediatric surgical patients was relatively high (26.2\%). Especially, the postoperative phase is the most critical time for the occurrence of PRAEs and desaturation was the predominant adverse event. Age less than a year, presence of upper respiratory tract infections, presence of secretions in the upper airways, ASA $\geq 3$ and airway related procedures were associated with PRAEs. Clinicians should carry out effective risk assessment, optimization and adequate preparation for the management of perioperative respiratory adverse events.

Keywords: Perioperative Respiratory Adverse Events, Perioperative complications, Pediatric Anesthesia General Anesthesia, 


\section{Introduction}

Adverse event is defined as unexpected and undesirable response to medication and medical intervention used to facilitate anesthesia and analgesia that threaten or cause patient injury or discomfort. Respiratory adverse events are any episodes of desaturation, partial or complete airway obstruction, persistent coughing, breath holding and bronchospasm (1-3). Despite improvements in pediatrics anesthesia, respiratory adverse events remain the most frequent cause of serious morbidity and mortality in the perioperative period. The three quarters of all critical incidents and the third of all perioperative cardiac arrests in pediatric anesthesia are caused by respiratory adverse events. Preoperative identification of those children at high risk is a challenging process $(4,5)$.

Pediatrics are venerable to those respiratory adverse events because they have hyper-reactive airway secondary to frequent upper respiratory tract infections (URTIs), high oxygen demands and low oxygen reserves. The most common respiratory adverse events under anesthesia are desaturation, breath holding, laryngospasm, bronchospasm and coughing. Among those events laryngospasm, bronchospasm and persistent hypoxemia could result potentially devastating complication and death (6-8).

Respiratory adverse events are inconsistently defined in much of studies making comparison difficult and results discrepancy on magnitude of the actual events. On the other way differences in medications and airway device used to manage respiratory adverse events could influence the outcome of the patient (1). Despite introduction of new monitoring modalities, pharmacological products and new clinical practice guidelines, the incidence of respiratory adverse events remained high and increase hospital stay more than two folds and increase the cost of medical care when compared to patients who didn't develop severe respiratory events $(1,9)$.

The patient's age, techniques of anesthesia induction, comorbidities and airway surgery were strongly associated with occurrence of adverse events during surgery. Occurrence of respiratory adverse events differs according to timing of extubation whether awake or deep plane of anesthesia, airway device and urgency of procedures are variable across studies and its complications are uncertain $(10,11)$ The severity of perioperative respiratory adverse events (PRAEs) and their complications depend on nature of surgery, presence comorbidity, early detection, and prompt corrective measures. The severity of the respiratory adverse event may range from transient damage with full recovery to unanticipated morbidity and mortality (2). 
Preoperative assessment and intervention are the key to successful management of the patient with upper respiratory infection, asthma and cardiac disease (12). Risk assessment in the preoperative period is recognized as very important to decrease respiratory events but still patient identification and selection is a real problem. If effective measures are not taken timely, serious respiratory complications and death would prevail (13). Critical respiratory incident recording and reporting system is poor particularly in the developing countries because fear of influence on their future carrier. Moreover, in limited resource countries electronic medical recording and reporting systems are not well established. These could have contribute for poor prevention PRAEs (31). In-contrast, in the developed world modern electronic based surveillances had significantly improved perioperative quality of care. Therefore, critical incident monitoring and reporting in anesthesia are very important for quality improvement and maintenance of high safety standards in anesthesia services (14).

The general objective of the current study was to assess the incidence and factors associated with perioperative respiratory adverse events (PRAEs) among pediatrics patients that underwent surgical operations under general anesthesia at University of Gondar and Tibebe-Ghion Comprehensive Specialized Hospitals, Northwest Ethiopia from March 1 to May 30, 2020. 


\section{Methods}

A Hospital-based cross-sectional follow-up study was conducted from March 1 to May 302020 at the University of Gondar and Tibebe-Ghion Comprehensive Specialized Hospitals. The Hospitals are located at Gondar and Bahirdar towns respectively in the Northwest Ethiopia.

The source population was all pediatric surgical patients $(0-12$ years of age $)$ that underwent surgical operations under general anesthesia and the study population was all pediatric surgical patients that underwent surgical operations under general anesthesia at UoGCSH and TGCSH during the study period.

All ASA I - V pediatric surgical patients of volunteer parents that underwent both emergency and elective surgical procedures were included in the study and those who had severe head injury, intubated patient, acute respiratory distress, hypoxia requiring mechanical ventilation in the preoperative period, operated for more than one time, and transferred to intensive care unit for mechanical ventilation after operation were excluded from the study.

The sample size was estimated by a single population proportion formula with $50 \%$ proportion, maximum acceptable difference $(\mathcal{E})=5 \%$, and $95 \%$ confidence interval:

$$
n=\left(Z \frac{\alpha}{2}\right)^{2} x p(1-p) / \varepsilon^{2}
$$

Where: $Z \alpha / 2=1.96$ and $p=50 \%=0.5$ and $\mathcal{E}=5 \%=0.05$ will be used so the sample size will be:

$$
\begin{gathered}
n=(1.96)^{2} x 0.5(1-0.5) / 0.05^{2} \\
n=385
\end{gathered}
$$

By reviewing the registration log, we found out that 780 and 660 pediatrics surgical procedures performed annually at UGCSH and TGCSH respective. So we used a correction formula to determine the corrected sample size.

$$
\begin{gathered}
n f=\frac{n}{1+n / N} \\
n f=\frac{385}{1+385 / 360} \\
n f=195
\end{gathered}
$$


We added $\mathbf{1 5 \%}$ for non-response rate and the final sample size was $\mathbf{2 2 5}$.

All eligible consecutive pediatric surgical patients who received general anesthesia were included in the study till the calculated sample size reached.

The dependent variable was perioperative respiratory adverse events which was measured in terms of any episode of either hypoxemia, coughing, breath holding, laryngospasm, or bronchospasm in the perioperative period. The independent variables were Patient factors (history of prematurity, age, ASA classification, family history of asthma, respiratory infections, and other comorbidities), anesthetic factors (techniques of anesthetic induction, type of airway device, number of intubation attempts, muscle relaxant, depth of anesthesia, perioperative opioid use, and experience of the anesthesia providers), and surgical factors (site of surgery, duration, and urgency of surgery).

Perioperative respiratory adverse event was measured as the occurrence of any episode of one or a combination of hypoxemia, coughing, breath holding, laryngospasm and bronchospasm in the perioperative period $(2,3,15,16,17,18-21)$. Operational definitions were prepared accordingly.

Laryngospasm: complete airway obstruction associated with muscle rigidity of the abdominal and chest walls and it need requirement of positive pressure ventilation or administration of succinylcholine (1).

Bronchospasm: increased respiratory effort, particularly during expiration and wheeze on auscultation or requires bronchodilators $(7,15)$.

Desaturation or hypoxemia: peripheral arterial oxyhemoglobin saturation $(\mathrm{SpO} 2)<95 \%$ more than 30 seconds measured by pulse oximetry regardless administrations of $100 \%$ Oxygen or $\mathrm{SpO} 2<90 \%$ in atmospheric air. The Oxygen saturation was recorded when the pulse oximetry showed consistent readings with no artifacts $(2,7)$.

Partial upper airway obstruction: the presence of airway obstruction in the combination with a snoring noise, an inspiratory stridor or respiratory efforts or paradoxical abdominal movement or both that can be effectively relieved by simple airway maneuvers (2).

Coughing: a series of pronounced, persistent coughs lasting more than 5 seconds (7).

Breath holding or apnea: if the patient had apnea more than 15 seconds or irregular breathing or if the apnea is associated with bradycardia or cyanosis (15).

Multiple intubation attempts: if required multiple intubation attempts more than 2 times (22). 
Active or current URTIs: the present of a minimum of two URTIs symptoms (rhinorrhea, sore or scratchy throat, sneezing, nasal congestion, malaise, cough, or fever, $\mathrm{T}>38^{\circ} \mathrm{C}$ ) at the time of surgery together with a confirmation by a parent (15).

Recent URTIs: the history of URTIs within 4 weeks before surgery but resolved at the time of surgery (15).

Acute respiratory distress: the presence of tachypnea, intercostal and subcostal retractions, nasal flaring, grunting, and cyanosis in room air and requires high flow Oxygen (23).

Light anesthesia: the presence of patient's movement with increased heart rate, blood pressure and respiratory rate change by more than $30 \%$ from baseline (24).

Oropharyngeal secretions: the presence of secretion that requires suctioning of more than once (25).

Pulmonary aspiration: a known or suspected inhalation of foreign material such as gastric contents into the respiratory tract associated with new or worsening respiratory signs such as hypoxia (26).

Airway related surgery: the surgical procedures involving the airway which includes ENT and maxillofacial procedures such as adinotonsilectomy, cleft palate repair, direct laryngoscopy, and bronchoscopy $(25,27,28)$.

Each complication was scored according to its severity from 1 (no complication) to 4 (most serious complication). For patients who received anesthesia with endotracheal tubes or laryngeal mask airways, scores were multiplied by a constant factor of five, and for those with face-masks scores were multiplied by a constant factor of three. Depending on the airway device used, patients therefore received a composite score of 5 - 20 for ETT or LMA and 3 - 12 face-mask for each adverse event. The scoring system for complications is based on a previously published scale and is described [Table 1] (25).

Respiratory adverse events were recorded starting from induction to the first postoperative hour. A patient experiencing PRAEs was counted just once independent of the numbers of events in perioperative period. Data was collected by using a pre-tested structured questionnaire. The questionnaire was primarily prepared in English language and translated to Amharic language. Training for data collectors and supervisors was provided by the principal investigator. A pilot was conducted on $20(8 \%)$ patients who were not included in the main study. Then necessary corrections were made accordingly to the questionnaire for the main study. The data collectors and supervisors were closely mentored by the principal investigator throughout the study period. The data collectors directly observed the children and reviewed their charts to collect the necessary data. The collected data was checked for the completeness and clarity. 
After completion of data collection, the variables were coded and cleaned. The data was entered into the Epi-data software (version 4.2) for cleaning for errors and was analyzed by SPSS version 20 (IBM Corporate). The normality of the data was tested by using Shapiro-Wilk normality test. Descriptive statistic was done and presented with frequency, percentage, mean, standard deviation, median and inter-quartile range (IQR). The relationships of nominal data with postoperative pain were analyzed by cross-tabulations using the Chi-squared or Fisher's exact tests as appropriate. Hosmer-Lemeshow test was used to assess the goodness of fit. The variance inflation factors and tolerance were used to diagnose multicollinearity. The associations between the independent variables and outcome variable were determined at $95 \%$ confidence interval with chi-squared test, bivariate and multivariate binary logistic regression. The cut-point of statistical significance was $\mathrm{p}<0.2$ for bivariate and $<0.05$ for multivariate regression. The crude and adjusted Odds Ratio were used to see the strength of the association for bivariate and multivariate logistic regression respectively. 


\section{Result}

A total of 210 pediatric surgical patients who received general anesthesia were included in this study with a $93.3 \%$ response rate. The remaining data from 15 patients was excluded due to incompleteness. The majority of the patients $115(54.8 \%)$ were males. The median age of the participants was $4.0(1.1-8.0)$ years [Table 2]. The majority of the patients were classified under ASA 1 and 2 which were 189 (90\%) patients while the rest were ASA 3 and above. The 23 (11\%) patients had URTIs. Eighty-nine (42.4\%) patients had low hemoglobin levels $(<11 \mathrm{~g} / \mathrm{dl})$ preoperative. The mean duration of surgery was $82 \pm 41.5$ minutes [Table 3].

The overall incidence of PRAEs among pediatric surgical patients who underwent surgery under general anesthesia at UOGCSH and TGCSH was found to be 55 (26.2\%, 95\% CI: 20.5\%, 30.9\%). We have observed a total of 129 episodes of PRAEs. Desaturation was found to be the predominant adverse event which occurred $61(47.3 \%)$ times; followed by partial upper airway obstruction 21 (16.3\%), breath holding $17(13.2 \%)$, persistent coughing 15 (11.6\%), laryngospasm $11(8.5 \%)$ and bronchospasm 2 (1.6\%). Most of the adverse events $89(69.0 \%)$ were occurred during the postoperative period while 23 (17.8) were occurred during induction of anesthesia, and $17(13.2 \%)$ during the maintenance phase [Figure 1].

Regarding severity of PRAEs, $10(4.8 \%)$ patients had serious desaturation $(\mathrm{SpO} 2<80 \%)$ and $1(0.5 \%)$ patient had continuous coughing, 3 (1.4\%) patients had complete airway obstruction which required muscle relaxant, and 9 (4.3\%) patients had laryngospasm which was effectively managed by the applications of simple airway maneuvers and positive airway pressure [Table 5].

Among four pediatric surgical patients who had family history of asthma, bronchospasm was occurred in the two patients. One was during induction and the other was after extubation. Fisher-exact test has showed the significant association between family history of asthma and perioperative bronchospasm. The relations of independent variables with outcome variables in Pearson's Chi-squared and Fisher-exact tests are presented [Table 4]. In the bivariate binary logistic regression analysis, comorbidity, emergency surgery, multiple attempts of tracheal intubation ( $\geq 3$ attempts), light anesthesia, perioperative opioid use and duration of surgery $\geq 60$ minutes were found associated with PRAEs. The final analysis model (multivariate binary logistic regression) demonstrated that age less than a year, presence URTIs (recent or active), presence of secretions in the upper airways, $\mathrm{ASA} \geq 3$ and airway related procedures were associated with PRAEs. 
Among pediatric surgical patients, those who had URTIs were more than 7 times likely to develop PRAEs (AOR: 7.6, 95\% CI: 1.9, 30.2, p: 0.004). The likelihood of PRAEs to occur in infants was 3.6 times than older pediatric surgical patients (AOR: 3.6, 95\% CI: 1.3, 10.0, p: 0.012). Interestingly, repeated attempts of tracheal intubation were noticed among infants (66.6\% vs. 33.3\%, p: 0.008). ASA physical status of 3 and above increases the occurrence of PRAEs by more than 5 folds (AOR: 5.2, 95\% CI: 1.9, 22.9, p: 0.029). Pediatric surgical patients who underwent airway related procedures had developed PRAEs more frequently compared to their counterparts (AOR: 6.0,95\% CI: 1.5, 24.1, p: 0.012). Having moderate to copious oropharyngeal secretions was found associated with the occurrence of PRAEs (AOR: 4.8, 95\% CI: 1.4, 15.9, p: 0.011). The results of this study claimed that prematurity, premedication, induction agents, types of airway devices and experiences of the anesthetists were not associated with PRAEs [Table 6]. 


\section{Discussion}

Perioperative respiratory adverse events (PRAEs) are the most common critical incidents encountered in the practice of pediatric anesthesia. In this study, PRAEs were defined in terms of perioperative occurrence of one or more episodes of desaturation, breathe holding, persistent coughing, partial or complete upper airway obstruction, laryngospasm and bronchospasm $(1,2,29)$.

The overall incidence of PRAEs among pediatric surgical patients who underwent surgery under general anesthesia at UOGCSH and TGCSH was $26.2 \%$. This result was congruent with a study done by Mamie et al, in which, it was $21 \%$ (1). A systematic review also has revealed that the incidence of PRAEs was in the ranges of $8 \%$ to $21 \%$ (2). Our finding was relatively lower compared to the results presented by Ramgolam et al. which was $26 \%-43 \%$ (7). The differences can be explained by the inclusion of pediatric surgical patients with high risk of developing PRAEs only in the previous study. Whereas, it was relatively higher when compared to an Australian study; in which, the overall incidence was 15\% (17). The variations might be due to that in the current study infants were included. We have found that there was an increased risk of developing PRAEs in infants compared to older children (42.3\% vs. 20.9\%, AOR: 3.6, 95 \% CI: 1.3, 10.0, p: 0.012). Our study has demonstrated that there was higher incidence of PRAEs compared to other studies; in which, PRAEs were occurred only among $2.8 \%, 5.7 \%$ and $17.8 \%$ pediatric surgical patients (3032). Higher incidence in our study might be due to the inclusion of patients with higher ASA physical status ( $\geq 3$ ). A retrospective multicenter study done in Thailand among 25,098 patients showed that the incidence of PRAEs was $4.6 \%$. The discrepancies might be explained by the incorporation of a large numbers of emergency patients in our study (54.3\% vs. $27.4 \%$ ) (13). In-addition, the differences in the study design and definitions of PRAEs might explain it.

Oxygen desaturation was found in 50 (23.8\%) patients which was the most common PRAEs. We found that desaturation was associated with most of patients who had URTIs and secretions. This was similar with multicenter study done in Thailand in terms of the overall occurrence but it was more frequent in the intraoperative period and lesser in the emergence phase of anesthesia (13). In this study, desaturation was found predominantly after tracheal extubation. This finding was supported by a Serbian study. Anesthetic agents are known for diminishing respiratory drive that can results in hypoxemia in the postoperative period (30).

Persistent coughing and upper airway obstruction were occurred $15(7.1 \%)$ and $21(10 \%)$ respectively. A systematic review pointed out the incidence of upper airway obstruction in the ranges between $3.6 \%$ and 
9\% (2). However, Budic and D. Simic have reported that persistent coughing was occurred only in 3 out of 682 patients. The differences in definitions of persistent coughing might cause the inconsistencies of results. In the current study, persistent coughing was defined as coughing that sustained for 5 seconds; however, others have defined it as coughing that sustained for $5-10$ seconds $(33,34)$.

In $16(7.6 \%)$ patients, breathe holding was found and it was higher compared to a study done by Tait et al (25). A large numbers of infant patients (52 (24.8\%)) was included in our study. Younger children have a tidal volume that occurs at the same volume as a closing volume; therefore, the terminal bronchioles close easily causing apnea and desaturation (30). Neuromuscular blocking agents were used extensively in 120 (57.1\%) patients. In our study areas, there was no a qualitative monitoring of residual neuromuscular blockade. This might contribute for higher incidence of breath holding among our patients (35).

The incidence of laryngospasm was found in $12(5.7 \%)$. It was congruent with a systematic review done by Regli et al, which claimed that the incidence of laryngospasm could ranges from $0.1 \%$ to $16 \%$. Particularly in general pediatrics population, it was 4\% (2). The commonest time of occurrence was the extubation period. In current study laryngospasm was associated with presence of secretions and URTIs. This result was similar with the previous studies conducted in Ethiopia $(24,36)$. The presence of family history of asthma was associated with an increased risk of bronchospasm in the perioperative period. This is compatible with the study done by Jackson and colleagues. The phenomenon can be explained by a genetically heightened bronchial hyper-responsiveness in children born from parents having asthma (37).

Among patient presented with URTIs, the incidence of PRAEs was 17 (73.9\%). In high-risk patients, the incidence could be as high as $50 \%(2,15,38)$. The reason for higher occurrence in our study might be the use of invasive airway devices (endotracheal tubes) in the majority of patients 15 (65.2\%) who had URTIs (2, $28,31)$. Our results tend to demonstrate the advantages of LMA (17.4) and FM (25) over ETT (29.4\%) among but no statistically significant associations obtained. Similarly with a study done in Serbia and Montenegro, the patients that presented with recent or current sign and symptoms of URTIs were found to have increased risk of developing PRAEs (AOR: 7.6, 95\% CI: 1.9, 30.2, p: 0.004). The higher risk of PRAEs during URTIs can be justified by the morphologic damage to the respiratory epithelium and mucosa after infections and sensitize the airway to potentially irritant anesthetic gases and secretions that results in activations of irritant receptors and contractions of smooth muscle of the airway (30). A study has showed that there was increased incidence of PRAEs in patients with an active URTIs than those who had recent URTIs ( 2 - 4 weeks before the procedure) (39). The effects of URTIs on the airway by the infections could last for several weeks (3). In 
our study there was no significant difference in the occurrence of PRAEs whether the URTI is recent or active (84.6\% vs. $60 \%$, p: 0.34). The incidence of PRAEs was significantly higher immediately after tracheal extubation than during induction of anesthesia and the intraoperative phase (30).

Harmoniously with other studies, infants were more vulnerable to develop PRAEs than older patients (AOR: 3.6, 95\% CI: 1.3, 10.0, p: 0.012) $(8,20)$. Desaturation occurs more frequently in infants and it was supported by Nigerian and Australian studies $(5,40)$. Infants have higher oxygen demand and low oxygen reserves which make them risky to PRAEs. In-addition, breath holding was common in this group of patients due to immature the respiratory center in the central nervous system. We have noticed that multiple attempts of tracheal intubation in infants than older patients (66.7\% vs. 33.3\%, p: 0.008). A study done in Thailand concluded that multiple intubation attempts were associated with PRAEs (22).

Among patients with higher ASA physical status $(\geq 3)$, higher rates of PRAEs compared to those with lower ASA physical status (AOR: 5.2, 95\% CI: 1.2, 22.9, p: 0.029). Previous studies also have showed the strong association between higher ASA physical status and PRAEs (4, 13). In developing countries, anesthetists frequently encounter poorly treated comorbidities which could lead to the occurrence of PRAEs $(4,5,41)$. The majority of the patients in our study have undergone emergency surgical operations $114(54 \%)$; therefore, poor optimization and limited availability of preoperative investigation modalities might contribute for higher incidence of PRAEs (41). We have observed that patients who had low levels of hemoglobin had similar incidence of PRAEs compared to normal hemoglobin levels.

The risk of developing PRAEs was increased by 6-folds when a surgical procedure involves the airway (AOR: 6.0, 95\% CI: 1.5, 24.1, p: 0.012). Airway related procedures were associated with frequent desaturation and upper airway obstruction. Our finding was supported by previous multicenter prospective studies conducted in Europe and USA $(3,5,42)$. Children who come for airway related procedures commonly have chronic airway inflammation and bronchial hypersensitivity that could result in PRAEs more frequently. Direct surgical instrumentation and manipulation of the airway can lead to laryngeal reflex response (2).

The presence of moderate to copious oropharyngeal secretions was found associated with PRAEs, particularly with desaturation and partial upper airway obstruction. Pediatrics surgical patients who had oropharyngeal secretions that required suctioning more than once were nearly 5 times more likely to sustain PRAEs (AOR: 4.8, 95\% CI: 1.4, 15.9, p: 0.011). Soiling of the airway which necessitate repeated suctioning can potentiate airway hyper-reactivity (25). 


\section{Strengths and limitations}

The study was the first for its type in the study areas and the country at large. It can be used as a cornerstone for future studies in the field. However, the study did not measure hypoventilation and residual neuromuscular blockade due to the lack of the devices in the post-anesthesia care units of the hospitals. As a cross-sectional study, it cannot determine cause and effect relations. 


\section{Conclusions}

The overall incidence of perioperative respiratory adverse events (PRAEs) among pediatric surgical patients was relatively high (26.2\%). Especially, the postoperative phase is the most critical time for the occurrence of PRAEs and desaturation was the leading adverse event. Age less than a year, presence URTIs (recent or active), presence of secretions in the upper airways, ASA $\geq 3$ and airway related procedures were associated with PRAEs. Clinicians should carry out effective risk assessment, optimization and adequate preparation for the management of perioperative respiratory adverse events. 


\section{Declarations}

\section{Acknowledgements}

None

\section{Funding}

College of Medicine and Health Sciences, University of Gondar, Gondar, Ethiopia.

Felege-Hiwot Referral Hospital, Bahirdar, Amhara National Regional State, Ethiopia.

\section{Availability of data and materials}

The datasets generated and/or analysed during the current study are not publicly available due to security and confidentiality issues but are available from the corresponding author on reasonable request.

\section{Authors' contributions}

DMW and YBW have conceptualized the study and objectives. DMW has developed the proposal. YBW, WBC, HA and MMW have criticized the proposal. All the authors had participated in the data and statistical analyses. DMW and YBW have led the manuscript preparation. All the authors read and approved the final manuscript.

\section{Ethics approval and consent to participate}

Ethical clearance to conduct the research was obtained from the Ethical Review Board of School of Medicine, College of Medicine and Health Sciences, University of Gondar. Written informed consent forms in Amharic and English languages were presented. Informed consent was taken from a legal care-giver of each patient after brief explanation. Every participant was allowed to discontinue participation if did not want to finish it. Also the participants were assured that their treatment and other benefits they can gain from the hospitals will not be interrupted due to their withdrawal. Confidentiality was ensured by removing identifiers and locking the questionnaires after data collection in a secured area. Additionally, when the patients found experiencing clinically significant perioperative respiratory adverse events, the data collectors had reported for the corresponding clinicians (Anesthetist, Surgeon or Nurse) to provide the appropriate management. All methods were carried out in accordance with relevant guidelines and regulations in the Ethical Declaration.

\section{Consent for publication}

Not applicable; this article does not include any personal or clinical details of any participant.

\section{Competing interests}

The authors declared that they have no competing interests. 


\section{List of Acronyms and Abbreviations}

$\begin{array}{ll}\text { AOR } & \text { Adjusted Odds Ratio } \\ \text { ASA } & \text { American Society of Anesthesiologist } \\ \text { GA } & \text { General Anesthesia } \\ \text { LMA } & \text { Laryngeal Mask Airways } \\ \text { PRAEs } & \text { Perioperative Respiratory Adverse Events } \\ \text { RCTs } & \text { Randomized Control Trials } \\ \text { SPO2 } & \text { Peripheral Oxygen Saturation } \\ \text { SPSS } & \text { Statistical Package for Social Science } \\ \text { TGCSH } & \text { Tibebe-Ghion Comprehensive Specialized Hospital } \\ \text { UOGCSH } & \text { University of Gondar Comprehensive and Specialized Hospital } \\ \text { URTIs } & \text { Upper Respiratory Tract Infections }\end{array}$




\section{References}

1. Mamie C, Habre W, Delhumeau C, Barazzone Argiroffo C, Morabia A. Incidence and risk factors of perioperative respiratory adverse events in children undergoing elective surgery. Pediatric Anesthesia. 2004;14(3):218-24.

2. Regli A, von Ungern-Sternberg BS. Diagnosis and management of respiratory adverse events in the operating room. Current Anesthesiology Reports. 2015;5(2):156-67.

3. von Ungern-Sternberg BS, Davies K, Hegarty M, Erb TO, Habre W. The effect of deep vs. awake extubation on respiratory complications in high-risk children undergoing adenotonsillectomy: a randomised controlled trial. European Journal of Anaesthesiology (EJA). 2013;30(9):529-36.

4. Lee S, Reddington E, Koutsogiannaki S, Hernandez MR, Odegard KC, DiNardo JA, et al. Incidence and risk factors for perioperative cardiovascular and respiratory adverse events in pediatric patients with congenital heart disease undergoing noncardiac procedures. Anesthesia \& Analgesia. 2018;127(3):724-9.

5. Murat I, Constant I, Maud'huy H. Perioperative anaesthetic morbidity in children: a database of 24165 anaesthetics over a 30-month period. Pediatric Anesthesia. 2004;14(2):158- 66.

6. Hackett S, Jones R, Kapila R. Anaesthesia for pneumonectomy. Bja Education. 2019;19(9):297-304.

7. Ramgolam A, Hall GL, Zhang G, Hegarty M, von Ungern-Sternberg BS. Inhalational versus Intravenous Induction of Anesthesia in Children with a High Risk of Perioperative Respiratory Adverse EventsA Randomized Controlled Trial. Anesthesiology: The Journal of the American Society of Anesthesiologists. 2018;128(6):1065-74.

8. Drake-Brockman TF, Ramgolam A, Zhang G, Hall GL, von Ungern-Sternberg BS. The effect of endotracheal tubes versus laryngeal mask airways on perioperative respiratory adverse events in infants: a randomised controlled trial. The Lancet. 2017;389(10070):701-8.

9. Lerman J. Perioperative respiratory complications in children. The Lancet. 2010;376(9743):745-6.

10. Najafi N, Veyckemans F, Vanhonacker D, Legrand C, Van de Velde A, Vandenplas Y, et al. Incidence and risk factors for adverse events during monitored anaesthesia care for gastrointestinal endoscopy in children: A prospective observational study. Eur J Anaesthesiol. 2019;36(6):390-9. 
11. Charuluxananan S, Suraseranivongse S, Jantorn P, Sriraj W, Chanchayanon T, Tanudsintum S, et al. Multicentered study of model of anesthesia related adverse events in Thailand by incident report (The Thai Anesthesia Incidents Monitoring Study) results. Medical journal of the Medical Association of Thailand. 2008;91(7):1011.

12. White MC, Barki BJ, Lerma SA, Couch SK, Alcorn D, Gillerman RG. A Prospective observational study of anesthesia-related adverse events and postoperative complications occurring during a surgical mission in Madagascar. Anesthesia \& Analgesia. 2018;127(2):506- 12.

13. Bunchungmongkol N, Somboonviboon W, Suraseranivongse S, Vasinanukorn M, Chau- in W, Hintong T. Pediatric anesthesia adverse events: the Thai Anesthesia Incidents Study (THAI Study) database of 25,098 cases. JOURNAL-MEDICAL ASSOCIATION OF THAILAND. 2007;90(10):2072.

14. De Graaff JC, Sarfo Mc, Van Wolfswinkel L, van der Werff DB, Schouten AN. Anesthesia-related critical incidents in the perioperative period in children; a proposal for an anesthesia-related reporting system for critical incidents in children. Pediatric Anesthesia. 2015;25(6):621-9.

15. von Ungern-Sternberg BS, Boda K, Schwab C, Sims C, Johnson C, Habre W. Laryngeal mask airway is associated with an increased incidence of adverse respiratory events in children with recent upper respiratory tract infections. Anesthesiology: The Journal of the American Society of Anesthesiologists. 2007;107(5):7149.

16. Mir ghassemi A, Neira V, Ufholz LA, Barrowman N, Mulla J, Bradbury CL, et al. A systematic review and meta-analysis of acute severe complications of pediatric anesthesia. Pediatric Anesthesia. 2015;25(11):1093-102.

17. von Ungern-Sternberg BS, Boda K, Chambers NA, Rebmann C, Johnson C, Sly PD, et al. Risk assessment for respiratory complications in paediatric anaesthesia: a prospective cohort study. The Lancet. 2010;376(9743):773-83.

18. Li L, Zhang Z, Yao Z, Wang H, Wang H, An H, et al. The impact of laryngeal mask versus other airways on perioperative respiratory adverse events in children: A systematic review and meta-analysis of randomized controlled trials. International Journal of Surgery. 2019;64:40-8. 
19. Terry KL, Disabato J, Krajicek M. Snoring, Trouble Breathing, Un-Refreshed (STBUR) screening questionnaire to reduce perioperative respiratory adverse events in pediatric surgical patients: a quality improvement project. AANA J. 2015;83(4):256-62.

20. Desalu I, Adeyemo W, Akintimoye M, Adepoju A. Airway and respiratory complications in children undergoing cleft lip and palate repair. Ghana medical journal. 2010;44(1).

21. Mason K, Green S, Piacevoli Q. Adverse event reporting tool to standardize the reporting and tracking of adverse events during procedural sedation: a consensus document from the World SIVA International Sedation Task Force. British journal of anaesthesia. 2012;108(1):13- 20.

22. Mandee S, Jitpakdee T, BNS TB, Aroonpruksakul N. The Incidence of Perioperative Adverse Events in Neonates and Infants Undergoing Non Cardiac Surgery with General Anesthesia. JOURNAL OF THE MEDICAL ASSOCIATION OF THAILAND. 2017;100(9):44.

23. Group PALICC. Pediatric acute respiratory distress syndrome: consensus recommendations from the Pediatric Acute Lung Injury Consensus Conference. Pediatric critical care medicine: a journal of the Society of Critical Care Medicine and the World Federation of Pediatric Intensive and Critical Care Societies. 2015;16(5):428.

24. Birlie Chekol W, Yaregal Melesse D. Incidence and Associated Factors of Laryngospasm among Pediatric Patients Who Underwent Surgery under General Anesthesia, in University of Gondar Compressive Specialized Hospital, Northwest Ethiopia, 2019: A Cross-Sectional Study. Anesthesiology Research and Practice. 2020;2020.

25. Tait AR, Malviya S, Voepel-Lewis T, Munro HM, Siewert M, Pandit UA. Risk factors for perioperative adverse respiratory events in children with upper respiratory tract infections. Anesthesiology: The Journal of the American Society of Anesthesiologists. 2001;95(2):299-306.

26. Katz SL, Monsour A, Barrowman N, Hoey L, Bromwich M, Momoli F, et al. Predictors of postoperative respiratory complications in children undergoing adenotonsillectomy. J Clin Sleep Med. 2020;16(1):41-8.

27. Luce V, Harkouk H, Brasher C, Michelet D, Hilly J, Maesani M, et al. Supraglottic airway devices vs tracheal intubation in children: a quantitative meta-analysis of respiratory complications. Pediatric Anesthesia. 2014;24(10):1088-98. 
28. Kim SY, Kim JM, Lee JH, Kang YR, Jeong SH, Koo B-N. Perioperative respiratory adverse events in children with active upper respiratory tract infection who received general anesthesia through an orotracheal tube and inhalation agents. Korean journal of anesthesiology. 2013;65(2):136.

29. El-Metainy S, Ghoneim T, Aridae E, Abdel Wahab M. Incidence of perioperative adverse events in obese children undergoing elective general surgery. British journal of anaesthesia. 2010;106(3):359-63.

30. Budić I, Simić D. Risk factors for respiratory adverse events during general anesthesia in children. Emergency. 2004;11(3):118-22

31. Lee LK, Bernardo MKL, Grogan TR, Elashoff DA, Ren WH. Perioperative respiratory adverse event risk assessment in children with upper respiratory tract infection: Validation of the COLDS score. Pediatric Anesthesia. 2018;28(11):1007-14.

32. Subramanyam R, Yeramaneni S, Hossain MM, Anneken AM, Varughese AM. Perioperative respiratory adverse events in pediatric ambulatory anesthesia: development and validation of a risk prediction tool. Anesthesia \& analgesia. 2016;122(5):1578-85.

33. Gharaei B, Aghamohammadi H, Jafari A, Razavi S, Kamranmanesh M, Kermany ASP. Use of laryngeal mask airway in children with upper respiratory tract infection, compared with face mask: randomized, single blind, clinical trial. Acta Anaesthesiologica Taiwanica. 2011;49(4):136-40.

34. de Carvalho ALR, Vital RB, de Lira CCS, Magro IB, Sato PTS, Lima LHN, et al. Laryngeal Mask Airway Versus Other Airway Devices for Anesthesia in Children With an Upper Respiratory Tract Infection: A Systematic Review and Meta-analysis of Respiratory Complications. Anesthesia \& Analgesia. 2018;127(4):941-50.

35. Murphy GS, Szokol JW, Marymont JH, Greenberg SB, Avram MJ, Vender JS. Residual neuromuscular blockade and critical respiratory events in the postanesthesia care unit. Anesthesia \& Analgesia. 2008;107(1):130-7.

36. Haile M, Legesse S, Miressa S, Desalegn N. Magnitude and Associated Risk Factors of Perioperative Pediatrics Laryngospasm under General Anesthesia. InternMed. 2015;5(203):2.

37. Jackson O, Basta M, Sonnad S, Stricker P, LaRossa D, Fiadjoe J. Perioperative risk factors for adverse airway events in patients undergoing cleft palate repair. The Cleft Palate- Craniofacial Journal. 2013;50(3):330-6. 
38. Huang H, Fang X. Effect of endotracheal intubation and laryngeal mask airway on perioperative respiratory adverse events in children with upper airway infections. Zhonghua yi xue za zhi. 2013;93(45):3626-8.

39. Li C, Wang D, Cheng T, Zheng X. Effects of recent upper respiratory-tract infections on incidence of the perioperative respiratory adverse events in children: a prospective cohort study. Beijing da xue xue bao Yi xue ban= Journal of Peking University Health sciences. 2017;49(5):814-8.

40. BPhil TFD-B. A randomised controlled trial comparing the impact of endotracheal tubes vs laryngeal mask airways on perioperative respiratory adverse events in infants.

41. Amucheazi AO, Ajuzieogu OV. Critical incidents during anesthesia in a developing country: A retrospective audit. Anesthesia, essays and researches. 2010;4(2):64-8.

42. Virag K, Sabourdin N, Thomas M, Veyckemans F, Habre W. Epidemiology and incidence of severe respiratory critical events in ear, nose and throat surgery in children in Europe: A prospective multi-center observational study. European Journal of Anaesthesiology (EJA). 2019;36(3):185-93. 
Figures

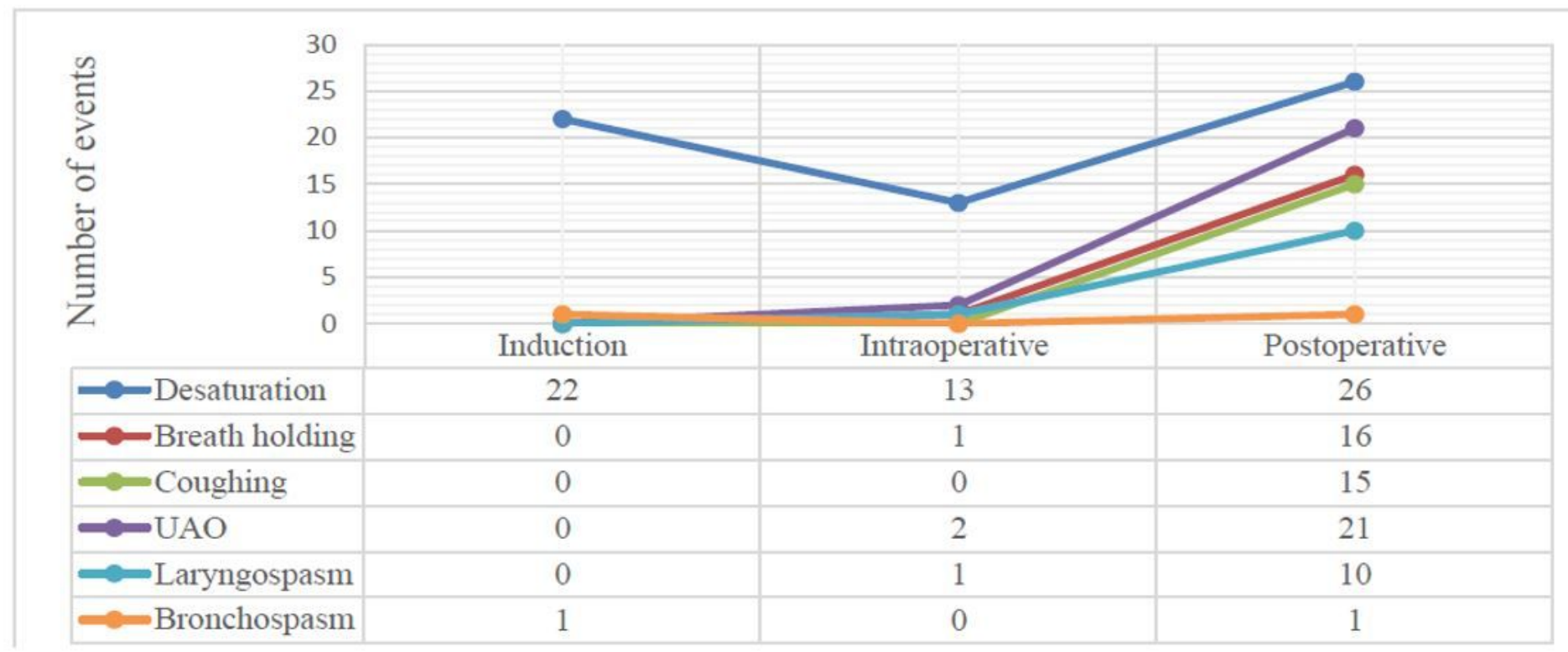

\section{Figure 1}

Line graphs showing the occurrences of perioperative respiratory adverse events among pediatrics surgical patients at UoGCSH and TGCSH, Northwest Ethiopia; March 1 - May 30, 2020 ( $N=210)$. 